\title{
On $\bar{\lambda}$-Statistically Convergent Double Sequences of Fuzzy Numbers
}

\author{
Ekrem Savaş \\ Department of Mathematics, Istanbul Ticaret University, Uskudar 36472, Istanbul, Turkey \\ Correspondence should be addressed to Ekrem Savaş, ekremsavas@yahoo.com \\ Received 17 July 2007; Accepted 13 December 2007 \\ Recommended by Jewgeni H. Dshalalow \\ We study the notion of $\bar{\lambda}$-statistically convergent for double sequence of fuzzy numbers and also \\ get some inclusion relations. \\ Copyright (C) 2008 Ekrem Savaş. This is an open access article distributed under the Creative \\ Commons Attribution License, which permits unrestricted use, distribution, and reproduction in \\ any medium, provided the original work is properly cited.
}

\section{Introduction}

Nanda [1] studied sequence of fuzzy numbers and showed that the set of all convergent sequences of fuzzy numbers form a complete metric space. Nuray [2] proved the inclusion relations between the set of statistically convergent and lacunary statistically convergent sequences of fuzzy numbers. Kwon and Shim [3] studied statistical convergence and lacunary statistical convergence of sequences of fuzzy numbers, and they showed that Nuray's conditions are sufficient as well as necessary. Savaş [4] introduced and discussed double convergent sequence of fuzzy numbers and showed that the set of all double convergent sequences of fuzzy numbers is complete. In [5], Savaş generalized the statistical convergence by using de la Vallee-Poussin mean. Quite recently, Savaş and Mursaleen [6] introduced of statistically convergent and statistically Cauchy for double sequence of fuzzy numbers.

In this paper, we continue to study the concepts of strongly double $[V, \bar{\lambda}]$-summable and double $S_{\bar{\lambda}}$-convergent for double sequence of fuzzy numbers.

\section{Preliminaries}

Before continuing with the discussion, we pause to establish some notation. Let $C\left(R^{n}\right)=\{A \subset$ $R^{n}$ : A compact and convex $\}$. The spaces $C\left(R^{n}\right)$ have a linear structure induced by the operations

$$
\begin{gathered}
A+B=\{a+b, a \in A, b \in B\}, \\
\lambda A=\{\lambda a, \lambda \in A\}
\end{gathered}
$$


for $A, B \in C\left(R^{n}\right)$, and $\lambda \in R$. The Hausdorff distance between $A$ and $B$ of $C\left(R^{n}\right)$ is defined as

$$
\delta_{\infty}(A, B)=\max \left\{\sup _{a \in A} \inf \|a-b\|, \sup _{b \in B} \inf _{a \in A}\|a-b\|\right\} .
$$

It is well known that $\left(C\left(R^{n}\right), \delta_{\infty}\right)$ is a complete (not separable) metric space.

A fuzzy number is a function $X$ from $R^{n}$ to $[0,1]$ satisfying

(1) $X$ is normal, that is, there exists an $x_{0} \in R^{n}$ such that $X\left(x_{0}\right)=1$;

(2) $X$ is fuzzy convex, that is, for any $x, y \in R^{n}$ and $0 \leq \lambda \leq 1$,

$$
X(\lambda x+(1-\lambda) y) \geq \min \{X(x), X(y)\}
$$

(3) $X$ is upper semicontinuous;

(4) the closure of $\left\{x \in R^{n}: X(x)>0\right\}$, denoted by $X^{0}$, is compact.

These properties imply that for each $0<\alpha \leq 1$, the $\alpha$-level set

$$
X^{\alpha}=\left\{x \in R^{n}: X(x) \geq \alpha\right\}
$$

is a nonempty compact convex, subset of $R^{n}$, as is the support $X^{0}$. Let $L\left(R^{n}\right)$ denote the set of all fuzzy numbers. The linear structure of $L\left(R^{n}\right)$ induces addition $X+Y$ and scalar multiplication $\lambda X, \lambda \in R$, in terms of $\alpha$-level sets by

$$
\begin{gathered}
{[X+Y]^{\alpha}=[X]^{\alpha}+[Y]^{\alpha},} \\
{[\lambda X]^{\alpha}=\lambda[X]^{\alpha}}
\end{gathered}
$$

for each $0 \leq \alpha \leq 1$.

Define for each $1 \leq q<\infty$,

$$
d_{q}(X, Y)=\left\{\int_{0}^{1} \delta_{\infty}\left(X^{\alpha}, Y^{\alpha}\right)^{q} d \alpha\right\}^{1 / q}
$$

and $d_{\infty}=\sup _{0 \leq \alpha \leq 1} \delta_{\infty}\left(X^{\alpha}, Y^{\alpha}\right)$. Clearly, $d_{\infty}(X, Y)=\lim _{q \rightarrow \infty} d_{q}(X, Y)$ with $d_{q} \leq d_{r}$ if $q \leq r$. Moreover, $d_{q}$ is a complete, separable, and locally compact metric space [7].

Throughout the paper, $d$ will denote $d^{q}$ with $1 \leq q \leq \infty$.

We will need the following definitions.

Definition 2.1. A double sequence $X=\left(X_{k l}\right)$ of fuzzy numbers is said to be convergent in the Pringsheim's sense or $P$-convergent to a fuzzy number $X_{0}$ if for every $\varepsilon>0$, there exists $N \in \mathcal{N}$ such that

$$
d\left(X_{k l}, X_{0}\right)<\epsilon \text { for } k, l>N \text {, }
$$

and we denote $P-\lim X=X_{0}$. The number $X_{0}$ is called the Pringsheim limit of $X_{k l}$.

More exactly, we say that a double sequence $\left(X_{k l}\right)$ converges to a finite number $X_{0}$ if $X_{k l}$ tend to $X_{0}$ as both $k$ and $l$ tends to $\infty$ independently of one another. 
Let $c^{2}(F)$ denote the set of all double convergent sequences of fuzzy numbers.

Definition 2.2. A double sequence $X=\left(X_{k l}\right)$ of fuzzy numbers is bounded if there exists a positive number $M$ such that $d\left(X_{k l}, X_{0}\right)<M$ for all $k$ and $l$,

$$
\|x\|_{(\infty, 2)}=\sup _{k, l} d\left(X_{k l}, X_{0}\right)<\infty .
$$

We will denote the set of all bounded double sequences by $l_{\infty}^{2}(F)$.

Let $K \subseteq \mathcal{N} \times \mathcal{N}$ be a two-dimensional set of positive integers and let $K_{m, n}$ be the numbers of $(i, j)$ in $K$ such that $i \leq n$ and $j \leq m$. Then the lower asymptotic density of $K$ is defined as

$$
P-\liminf _{m, n} \frac{K_{m, n}}{m n}=\delta_{2}(K) .
$$

In the case when the sequence $\left(K_{m, n} / m n\right)_{m, n=1,1}^{\infty, \infty}$ has a limit, then we say that $K$ has a natural density and is defined as

$$
P-\lim _{m, n} \frac{K_{m, n}}{m n}=\delta_{2}(K) .
$$

For example, let $K=\left\{\left(i^{2}, j^{2}\right): i, j \in \mathcal{N}\right.$, where $\mathcal{N}$ is the set of natural numbers. Then

$$
\delta_{2}(K)=P-\lim _{m, n} \frac{K_{m, n}}{m n} \leq P-\lim _{m, n} \frac{\sqrt{m} \sqrt{n}}{m n}=0
$$

(i.e., the set $K$ has double natural density zero).

Definition 2.3. A double sequence $X=\left(X_{k l}\right)$ of fuzzy numbers is said to be statistically convergent to $X_{0}$ provided that for each $\varepsilon>0$,

$$
P-\lim _{m, n} \frac{1}{n m}\left|\left\{(j, k) ; j \leq m, k \leq n: d\left(X_{k l}, X_{0}\right) \geq \epsilon\right\}\right|=0 .
$$

In this case, we write $s_{2}-\lim _{k, l} X_{k, l}=X_{0}$ and we denote the set of all double statistically convergent sequences of fuzzy numbers by $\operatorname{st}^{2}(F)$.

Definition 2.4. $\lambda=\left(\lambda_{n}\right)$ and $\mu=\left(\mu_{m}\right)$ could be two nondecreasing sequences of positive real numbers such that each tends to $\infty$ and

$$
\begin{array}{cc}
\lambda_{n+1} \leq \lambda_{n}+1, & \lambda_{1}=1, \\
\mu_{m+1} \leq \mu_{m}+1, & \mu_{1}=1 .
\end{array}
$$

A double sequence $X=\left(X_{k l}\right)$ of fuzzy numbers is said to be $\bar{\lambda}$-summable if there is fuzzy number $X_{0}$ such that

$$
P-\lim _{n m} \frac{1}{\bar{\lambda}_{n m}} \sum_{k \in I_{n}} \sum_{l \in I_{m}} d\left(X_{k l}, X_{0}\right)=0,
$$

where $I_{n}=\left[n-\lambda_{n}+1, n\right], I_{m}=\left[m-\mu_{m}+1, m\right]$, and $\bar{\lambda}_{n m}=\lambda_{n} \mu_{m}$. 
In this case, we say that $X$ is strongly double $\bar{\lambda}$-summable to $X_{0}$ and we denote the set of all strongly double $\bar{\lambda}$-summable sequences by $[V, \bar{\lambda}](F)$. If $\bar{\lambda}_{n m}=n m$, then strongly double $\bar{\lambda}$-summable reduces to $[C, 1,1](F)$, the space of strongly double Cesàro summable sequences defined as follows:

$$
P-\lim _{n m} \frac{1}{n m} \sum_{k, l=1,1}^{m n} d\left(X_{k l}, X_{0}\right)=0
$$

Definition 2.5. A double sequence $X=\left(X_{k l}\right)$ of fuzzy numbers is said to be double $\bar{\lambda}$-statistically convergent or $S_{\bar{\lambda}}$-convergent to $X_{0}$ if for every $\epsilon>0$,

$$
P-\lim _{n, m} \frac{1}{\bar{\lambda}_{n m}}\left|\left\{k \in I_{n}, l \in I_{m}: d\left(X_{k l}, X_{0}\right) \geq \epsilon\right\}\right|=0
$$

In this case, we write $S_{\bar{\lambda}}-\lim X=X_{0}$ or $X_{k l} \stackrel{P}{\rightarrow} X_{0}\left(S_{\bar{\lambda}}\right)$ and we denote the set of all double $S_{\bar{\lambda}}$-statistically convergent sequences of fuzzy numbers by $\left(S_{\bar{\lambda}}\right)(F)$.

If $\bar{\lambda}_{n m}=n m$, for all $n, m$, then the set $S_{\bar{\lambda}}(F)$ of $S_{\bar{\lambda}}$-convergent sequences reduces to the space $\operatorname{st}^{2}(F)$.

We need the following proposition in future. A metric $d$ on $L(\mathbb{R})$ is said to be a translation invariant if $d(X+Z, Y+Z)=d(X, Y)$ for $X, Y, Z \in L(\mathbb{R})$.

Proposition 2.6. If $d$ is a translation invariant metric on $L(\mathbb{R})$, then

$$
d(X+Y, 0) \leq d(X, 0)+d(Y, 0)
$$

Proof is clear so we omitted it.

In the next theorem, we give some connections between strongly double $\bar{\lambda}$-summable and double $\bar{\lambda}$-statistical convergences.

\section{Main results}

Theorem 3.1. A double sequence $X=\left(X_{k l}\right)$ of fuzzy numbers is strongly double $\bar{\lambda}$-summable $X_{0}$, then it is double $\bar{\lambda}$-statistically convergent to $X_{0}$.

Proof. Let $\epsilon>0$ and since

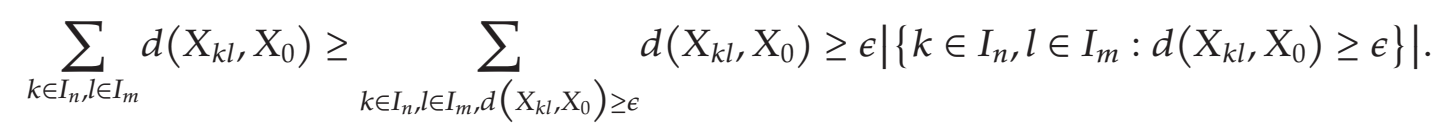

This implies that if a sequence $X=\left(X_{k l}\right)$ is strongly double $\bar{\lambda}$-summable $X_{0}$, then $X$ is double $\bar{\lambda}$-statistically convergent to $X_{0}$.

This completes the proof.

We have the following theorem.

Theorem 3.2. If a bounded $\left(X_{k l}\right)$ is double $\bar{\lambda}$-statistically convergent to $X_{0}$, then it is strongly double $\bar{\lambda}$-summable $\mathrm{X}_{0}$. 
Proof. Suppose that $\left(X_{k l}\right)$ is bounded and double $\bar{\lambda}$-statistically convergent to $X_{0}$. Since $X$ is bounded we write $d\left(X_{k l}, X_{0}\right) \leq M$ for all $k, l$. Also for given $\epsilon>0$ and $n$ and $m$ large we obtain

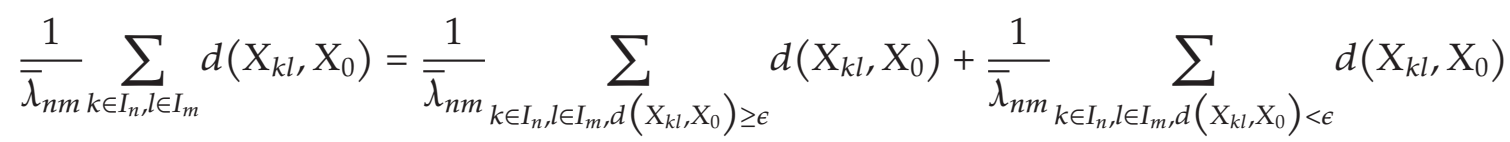

$$
\begin{aligned}
& \leq \frac{M}{\bar{\lambda}_{n m}}\left|\left\{k \in I_{n}, l \in I_{m}: d\left(X_{k l}, X_{0}\right) \geq \epsilon\right\}\right|+\epsilon,
\end{aligned}
$$

which implies that $X$ is strongly double $\bar{\lambda}$-summable $X_{0}$.

This completes the proof.

Theorem 3.3. If a sequence $X=\left(X_{k l}\right)$ of fuzzy numbers is double statistically convergent to $X_{0}$, then it is double $\bar{\lambda}$-statistically convergent to $X_{0}$ if and only if

$$
P-\lim _{n m} \inf \frac{\bar{\lambda}_{n m}}{n m}>0
$$

Proof. For given $\varepsilon>0$, we have

$$
\left\{k \leq n, l \leq m: d\left(X_{k l}, X_{0}\right) \geq \epsilon\right\} \supset\left\{k \in I_{n}, l \in I_{m}: d\left(X_{k l}, X_{0}\right) \geq \epsilon\right\}
$$

Therefore,

$$
\begin{aligned}
\frac{1}{n m}\left|\left\{k \leq n, l \leq m: d\left(X_{k l}, X_{0}\right) \geq \epsilon\right\}\right| & \geq \frac{1}{n m} \mid\left\{k \in I_{n}, l \in I_{m}: d\left(\left(X_{k l}, X_{0}\right) \geq \epsilon\right\} \mid\right. \\
& \geq \frac{\bar{\lambda}_{n m}}{n m} \frac{1}{\bar{\lambda}_{n m}} \mid\left\{k \in I_{n}, l \in I_{m}: d\left(\left(X_{k l}, X_{0}\right) \geq \epsilon\right\} \mid .\right.
\end{aligned}
$$

Taking the limit as $n, m \rightarrow \infty$ and using hypothesis, we get $X$ is double $\bar{\lambda}$-statistically convergent to $X_{0}$.

Conversely, suppose that $X \in \mathrm{st}_{2}(F)$ and since $\bar{\lambda}_{n m}=\lambda_{n} \mu_{m}$, either $P-\lim _{n} \inf \lambda_{n} / n=0$ or $P-\lim _{m} \inf \left(\mu_{m} / m\right)=0$ or both are zero. Then we can choose subsequences $(n(p))_{p=1}^{\infty}$ and $(m(q))_{q=1}^{\infty}$ such that $\lambda_{n_{(p)}} / n(p)<1 / p$ and $\mu_{m_{(q)}} / m(q)<1 / q$. Define a sequence $X=\left(X_{k l}\right)$ by

$$
X_{k l}= \begin{cases}1 & \text { if } k \in I_{n_{(p)}}, l \in I_{m(q)}(p, q=1,2, \ldots) \\ 0 & \text { otherwise. }\end{cases}
$$

Then $X \in[C, 1,1](F)$ and hence, by $\left[6\right.$, Theorem 6(a)], $X \in \mathrm{st}^{2}(F)$. But on the other hand, $X \notin[V, \bar{\lambda}](F)$ and from Theorem 3.1, $X \notin\left(S_{\bar{\lambda}}\right)(F)$; a contradiction and hence (3.3) must hold.

Finally, we conclude this paper by stating a definition which generalizes Definition 2.4 . 
Definition 3.4. Let $X=\left(X_{k l}\right)$ be a double sequence of fuzzy numbers and let $p$ be positive real numbers. The sequence $X$ is said to be strongly double $\bar{\lambda}_{p}$-summable if there is fuzzy number $X_{0}$ such that

$$
P-\lim _{n m} \frac{1}{\bar{\lambda}_{n m}} \sum_{k \in I_{n}} \sum_{l \in I_{m}} d\left(X_{k l}, X_{0}\right)^{p}=0 .
$$

In this case, we say that $X$ is strongly double $\bar{\lambda}_{p}$-summable to $X_{0}$. If $\bar{\lambda}_{n m}=n m$, then strongly double $\bar{\lambda}_{p}$-summable reduces to strongly double $p$-Cesàro summable to $X_{0}$.

Theorem 3.5. (1) Let $p \in(0, \infty)$. If a double sequence $X=\left(X_{k l}\right)$ of fuzzy numbers is strongly double $\bar{\lambda}_{p}$-summable $X_{0}$, then it is double $\bar{\lambda}$-statistically convergent to $X_{0}$.

(2) Let $p \in(0, \infty)$. If a bounded $\left(X_{k l}\right)$ is double $\bar{\lambda}$-statistically convergent to $X_{0}$, then it is strongly double $\bar{\lambda}_{p}$-summable $X_{0}$.

Proof. The proof of theorem is similar to that of Theorems 3.1 and 3.2 so we omitted it.

\section{References}

[1] S. Nanda, “On sequences of fuzzy numbers," Fuzzy Sets and Systems, vol. 33, no. 1, pp. 123-126, 1989.

[2] F. Nuray, "Lacunary statistical convergence of sequences of fuzzy numbers," Fuzzy Sets and Systems, vol. 99, no. 3, pp. 353-355, 1998.

[3] J. S. Kwon and H. T. Shim, "Remark on lacunary statistical convergence of fuzzy numbers," Fuzzy Sets and Systems, vol. 123, no. 1, pp. 85-88, 2001.

[4] E. Savaş, "A note on double sequences of fuzzy numbers," Turkish Journal of Mathematics, vol. 20, no. 2, pp. 175-178, 1996.

[5] E. Savaş, "On strongly $\lambda$-summable sequences of fuzzy numbers," Information Sciences, vol. 125, no. 1-4, pp. 181-186, 2000.

[6] E. Savaş and Mursaleen, "On statistically convergent double sequences of fuzzy numbers," Information Sciences, vol. 162, no. 3-4, pp. 183-192, 2004.

[7] P. Diamond and P. Kloeden, "Metric spaces of fuzzy sets," Fuzzy Sets and Systems, vol. 35, no. 2, pp. 241-249, 1990. 